\title{
Biota acuática en la Amazonia Peruana: diversidad y usos como indicadores ambientales en el Bajo Urubamba (Cusco - Ucayali)
}

\section{Aquatic biota in the Peruvian Amazon: diversity and uses as environmen- tal indicators in the lower Urubamba (Cusco - Ucayali)}

\author{
Hernán Ortega, Luisa Chocano, Carlos Palma e Iris Samanez
}

1 Departamento de Ictiología. Museo de Historia Natural. UniMersidad Nacionat Mayor de San varsidad Nacional Mayor de San Marcos (UNMSM). Av. Arenales 1256, Jesús María. Lima, Perú Email Hernán Ortega: hortega@terra.com.pe

Trabajo presentado a la XVIII Reunión Científica del Instituto de Investigaciones en Ciencias BiolóInvestigaciones en Ciencias Bioló-
gicas Antonio Raimondi, "200 años gicas Antonio Raimondi, "200 años del nacimiento de Charles Darwin y el 150 aniversario de la publicación de On the Origin of Species by Means of Natural Selection". Del 19 al 21 de agosto de 2009.

Publicado impreso: 20/10/2010 Publicado online: 29/09/2010

\section{Resumen}

En el presente trabajo se aplican índices biológicos de calidad ambiental y conservación, basados en el monitoreo biológico realizado entre año 2003 y 2009, en cinco localidades del río Bajo Urubamba. Fueron estudiadas las comunidades del plancton, bentos y peces. La diversidad del plancton comprendió 170 especies, basadas principalmente en Chlorophyta y Bacillariophyta. El bentos incluyó 112 especies, principalmente larvas y adultos de Arthropoda (Insecta). La diversidad de peces, incluye 176 especies, representadas por 26 familias y seis órdenes. El Índice Ephemeroptera + Plecoptera + Trichoptera (\%EPT), califico el área de estudio entre normal a muy buena calidad. El índice de Integridad Biológica (IBI) que determina el estado de conservación de los ambientes acuáticos, dio los mayores valores en Miaría y Sepahua. La elevada diversidad de las comunidades estudiados estaría relacionada a la heterogeneidad de hábitats y mayores recursos observados en la parte baja del área de estudio.

Palabras claves: comunidades biológicas, peces, riqueza, ambientes acuáticos, conservación

\section{Abstract}

In this paper we apply biological indices of environmental quality and conservation, based on biological monitoring conducted between 2003 and 2009 in five localities of the Bajo Urubamba River. Communities of plankton, benthos and fishes were studied. The diversity of plankton was 170 species, mainly based on Chlorophyta and Bacillariophyta. The benthos included 112 species, mainly larvae and adults of Arthropoda (Insecta). 176 fish species were recorded, represented by 26 families and six orders. The quality of the study area ranged from normal to very good, according to the index \%ETP. The conservation index of aquatic environments (IBI) gave the higher values for Miari and Sepahua. The high diversity of the communities studied could be related to the heterogeneity of habitats and greater resources than are observed in the lower part of the study area.

Palabras claves: biological communities, richness, fishes, aquatic habitats, conservation

\section{Introducción}

Los cuerpos de agua dulce constituyen un recurso natural invalorable en diversos aspectos: económico, cultural, estético, científico y educacional; en la actualidad, estos ecosistemas están experimentando cambios en su biodiversidad, calidad y cantidad por diversas actividades humanas que ocasionan contaminación, destrucción y degradación de hábitats.

La cuenca amazónica es el reservorio de la biota acuática más diversa del mundo, pero a su vez los cuerpos de agua dulce son ecosistemas muy sensibles y amenazados, y considerados en peligro en todo el mundo, por lo que se debe incidir en el conocimiento de su biodiversidad. Se reconoce que el conocimiento de la biodiversidad acuática amazónica en nuestras latitudes es incompleto (Dudgeon et al. 2005), y prioritario para cumplir los programas internacionales como los declarados por el Decenio Internacional para la Acción, "El agua, fuente de vida”, 20052015 de la UNESCO.

Uno de los grupos mejor conocidos son los peces, reconociéndose 4500 especies en las aguas neotropicales continentales (Lévêque et al. 2007); para las aguas continentales peruanas son registradas mil especies (Ortega \& Hidalgo 2008). Algunas zonas del Perú están mejor estudiadas, por ejemplo la ictiofauna del río Biabo (De Rham et al. 2001), el río Yavari (Ortega et al. 2003), el río Bajo Urubamba (Ortega et al., 2001) y la región sureste del Perú (Barthem et al. 2004, Goulding et al. 2004).

Otros grupos de los cuerpos de agua amazónicos estudiados son los del plancton de Madre de Dios (Samanez \& Zambrano 1995, Riofrio et al. 2003), microalgas de Pucallpa (Samanez
1979) y San Martín (Drouet et al. 1966), los rotíferos de Ucayali (Samanez 1988, Samanez \& Riofrio 1995, Samanez 2002) y Loreto (Samanez 1991).

También han contribuido al conocimiento de la biodiversidad en la cuenca Amazónica evaluaciones generales de biodiversidad en el río Camisea (Ortega 1996), en el Bajo Urubamba (Salcedo et al. 1998) y sobre temas de conservación (Ortega et al. 2007) y protocolos limnológicos (Ortega et al. 1998).

El presente trabajo informa los resultados del monitoreo biológico realizado entre el año 2003 y 2009, en las comunidades de plancton, bentos y peces, de cinco localidades del Bajo Urubamba, y aplica índices biológicos de calidad ambiental y conservación.

\section{Material y métodos Área de estudio}

El estudio comprendió ambientes acuáticos lóticos y lénticos de cinco localidades (Tabla 1) en el sistema del río Bajo Urubamba (Ucayali-Cusco). En cada localidad se establecieron tres estaciones de muestreo.

Tabla 1. Ubicación de las localidades de estudio en Coordenadas UTM .

\begin{tabular}{lcc}
\hline Localidades & $\mathbf{X}$ & $\mathbf{Y}$ \\
\hline Shivankoreni & 18L 0725843 & 8704492 \\
Kirigueti & 18L 0703749 & 8720403 \\
Miaría & 18L 0718774 & 8750414 \\
Sepahua & 18L 0713458 & 8766918 \\
Timpia & 18L 0737076 & 8663648 \\
\hline
\end{tabular}




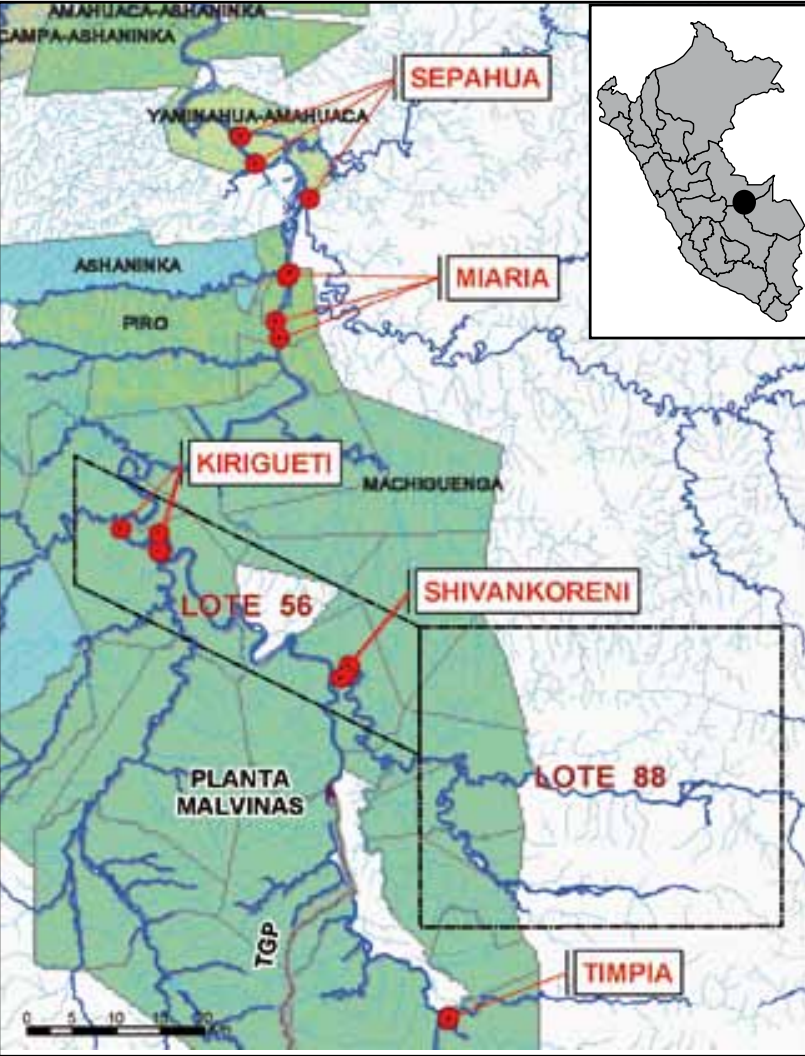

Figura 1. Cuenca del Bajo Urubamba y localidades de estudio. Cusco - Ucayali, Perú.

Shivankoreni: Ubicada en la margen derecha de los ríos Camisea y Urubamba. Estaciones: 1) a $300 \mathrm{~m}$ aguas arriba del puerto principal, 2) a $300 \mathrm{~m}$ aguas abajo y 3) a $500 \mathrm{~m}$ cerca de la desembocadura en el río Urubamba.

Kirigueti: Ubicada en el margen izquierdo del río Urubamba. Las estaciones: 1) tramo final del río Picha, 2) un brazo del río Urubamba, denominado "laguna temporal" porque parte del ańo se encuentra aislada y aun en creciente conserva su carácter léntico y 3) Quebrada Pitoniari, afluente en el margen izquierdo del río Urubamba, aguas abajo de la comunidad.

Miaría: Ubicada en el margen izquierdo del río Urubamba. Estaciones: 1) Quebrada Charapa, afluente de la margen izquierdo del río Urubamba 2) Quebrada Shimbillo, afluente de la margen derecha del río Urubamba y 3) río Miaría, tributario de la margen izquierda y evaluado cerca de su desembocadura en el río Urubamba.

Sepahua: Ubicada entre los márgenes derechos de los ríos Sepahua y Urubamba. Estaciones: 1) río Mishahua, afluente de la margen derecha del río Urubamba, aguas arriba de Sepahua; 2) río Sepahua, margen izquierda y 3) Quebrada Kumarillo, tributario del margen izquierdo del Urubamba, aguas arriba de Sepahua.

Timpia: Ubicada en el margen derecho del río Urubamba y comprende los ríos Timpia y Shihuaniro. Estaciones: 1) río Shihuaniro, sector final antes de su unión con el río Timpia 2) río Timpia, a $300 \mathrm{~m}$ antes de conectarse con el río Urubamba y 3) río Urubamba, márgenes del río o playas de la isla, frente al caserío de la comunidad.

En el campo se procedió a la descripción física y ecológica del hábitat, según Ortega et al. (1998), incluyendo el registro de parámetros fisicoquímicos básicos: oxigeno disuelto, temperatura, transparencia, $\mathrm{CO}_{2}, \mathrm{pH}, \mathrm{STD}$ y conductividad.

El plancton se colectó filtrando 20 litros de agua con una red estándar de 45 micras de abertura. La muestra fue fijada con formol $5 \%$.

Para el muestreo del bentos se usó la red Surber (marco metálico de $30 \times 30 \mathrm{~cm}$, malla de $1 \mathrm{~mm}$ ), colocándola en posición inversa a la corriente. Se realizaron tres réplicas en cada estación y la muestra fue fijada de inmediato con etanol $70 \%$.

Los peces se colectaron utilizando redes de arrastre a la orilla de 10 x $3 \mathrm{~m}$ y malla $5 \mathrm{~mm}$. La colecta se realizó efectuando cinco lances en cada estación. El material fue fijado directamente en una solución de formol $10 \%$ por 48 horas, pasando luego a una solución de etanol $70 \%$ y para su transporte en bolsas plásticas envueltas en gasa húmeda de etanol.

Cada muestra obtenida llevo adjunto los datos de campo propios y el análisis respectivo fue realizado en el Museo de Historia Natural de la UNMSM.

Las muestras fueron analizadas empleando un microscopio y estereoscopio. Las muestras de peces fueron separadas y lavadas por lotes, identificadas, preservadas en etanol $70 \%$, rotuladas, y depositadas en la Colección Ictiológica (MUSM). La clasificación siguió el orden según Reis et al. (2003) y Ortega \& Hidalgo (2008).

Para el análisis de la información biológica y determinación del estado de conservación de comunidades y ecosistemas acuáticos se emplearon los índices ecológicos: \%EPT (El Índice Ephemeroptera + Plecoptera + Trichoptera). Es la relación existente entre la cantidad de organismos que son indicadores de aguas limpias o de buena calidad, exigentes en altos valores de oxígeno. De acuerdo al porcentaje observado en las diferentes muestras de la presencia y magnitud de estos grupos indicadores se obtendrá una calificación del estado de conservación del ambiente acuático en estudio, según Roldan (1997)

IBI (Índice de Integridad Biológica). Sistema de calificación de hábitat diseñado por Karr (1981) para evaluar la condición de los cursos de agua en el hemisferio norte, adaptado a la composición y estructura de las poblaciones de peces amazónicos, y aplicado en una evaluación ictiológica entre Tarapoto (San Martín) y Yurimaguas (Loreto), de acuerdo con Ortega et al. (2007).

Se emplean consideraciones de Riqueza (criterio 1), número de especies registradas en cada localidad y la composición (criterios 2, 3 y 4) que involucra órdenes representativos (Characiformes, Siluriformes y Gymnotiformes). En los criterios 5 y 6 , presencia de peces periféricos (origen marino) y secundarios (tolerantes). En la categoría de estructura trófica (criterios 7, 8 y 9), presencia de peces omnívoros, detritívoros y carnívoros, respectivamente. Finalmente, la abundancia (criterio 10), número de ejemplares colectados, buena apariencia (criterio 11) y condición saludable de los peces (criterio 12).

\section{Resultados}

Derivados del monitoreo biológico que comprende 14 evaluaciones, desde septiembre 2003 hasta abril 2009.

\section{Descripción física de los ambientes acuáticos}

Agrupados por el tipo de agua y ambiente lótico, tenemos: 
Ríos de agua blanca: Urubamba, Timpia, Miaría, Mishahua y Sepahua. Presentan transparencia entre 0,02 y 0,15 m. Amplitud de cauce de 30 a $80 \mathrm{~m}$ y profundidad desde 0,4 a 4,5 m, entre vaciante y creciente. Orillas amplias, desprotegidas o con moderada cobertura vegetal. De sustrato duro: arcilla, arena, grava, canto rodado y rocas; presenta rápidos y remansos, como también micro hábitats formados por troncos sumergidos.

Ríos de agua clara: Camisea, Picha y Shihuaniro. Presentan transparencia entre $0,15 \mathrm{~m}$ y total; color verde claro a esmeralda, con variaciones entre la época lluviosa y seca, amplitud de cauce entre 15 y $70 \mathrm{~m}$, profundidad variable de 0,2 a $5 \mathrm{~m}$. Orillas de estrechas a amplias, desprotegidas o con cobertura vegetal moderada. De sustrato mixto, compuesto por arena, limo, canto rodado, piedras y rocas y, presenta hábitats formados por playas arenosas, remansos y rápidos.

Quebradas de agua clara: Kumarillo, Charapa, Pitoniari. Transparencia entre $0,05 \mathrm{~m}$ y total. Amplitud de cauce de 4 a $20 \mathrm{~m}$ y profundidad de 0,5 a $1,5 \mathrm{~m}$, entre vaciante y creciente. Con orillas amplias, desprotegidas, con escasa cobertura vegetal. De sustrato mixto, formado por canto rodado, grava, arena y limo, con hojarasca y mesohábitats formados por rápidos, pozas y vegetación sumergida.

Laguna temporal, brazo del río Urubamba, con agua blanca, transparencia entre 0,05 y $0,45 \mathrm{~m}$. Con amplitud de cauce de 27 a $80 \mathrm{~m}$, y profundidad entre 0,8 y $2,4 \mathrm{~m}$, entre vaciante y creciente. Presenta orillas moderadas, con cobertura vegetal herbácea y arbustiva. De sustrato mixto, con arena, limo y además de canto rodado; con hojarasca y vegetación sumergida.

Quebrada de agua blanca-clara: Shimbillo. Transparencia entre 0,05 y $0,50 \mathrm{~m}$. Amplitud de cauce de 4 a $10 \mathrm{~m}$ y profundidad de 0,3 a 2,5 m, entre vaciante y creciente. Presenta orillas estrechas, moderadamente protegidas, con cobertura vegetal de bosque secundario. Cauce con sustrato mixto, compuesto por canto rodado, grava, arena y limo, presenta micro hábitats formados por rápidos, pozas, troncos y vegetación sumergida.

La vegetación observada en las márgenes de los ríos fue de bosque ribereño compuesto principalmente por Tessaria integrifolia (pájaro bobo), Gynerium spp. (caña brava), Cecropia spp. (cetico) y Ficus spp. (ojé).

\section{Plancton}

El fitoplancton registró 170 especies reunidas en seis Divisiones. La mayor diversidad fue observada en Chlorophyta, seguido por Bacillariophyta y Cyanophyta, mientras que Rhodophyta, Pyrrhophyta y Chrysophyta presentaron valores mínimos (Tabla 2).

Tabla 2. Riqueza total del plancton por Division. Bajo Urubamba. Junio 2004 - abril 2009

\begin{tabular}{lc}
\multicolumn{1}{c}{ Division } & Riqueza total \\
\hline Bacillariophyta & 35 \\
Chrysophyta & 5 \\
Chlorophyta & 86 \\
Cyanophyta & 34 \\
Euglenophyta & 6 \\
Pyrrhophyta & 3 \\
Rodophyta & 1 \\
\hline \multicolumn{1}{c}{ Total } & $\mathbf{1 7 0}$ \\
\hline
\end{tabular}

Tabla 3. Riqueza (S) del plancton por Division y localidades. Bajo Urubamba. Junio 2004 - abril 2009.

\begin{tabular}{lccccc}
\hline & & & & \\
& & & & & \\
& & & & & \\
& & & & & \\
\hline Bacillariophyta & 25 & 33 & 21 & 26 & 35 \\
Chrysophyta & 3 & 0 & 2 & 2 & 1 \\
Chlorophyta & 50 & 49 & 39 & 42 & 37 \\
Cyanophyta & 21 & 25 & 27 & 24 & 27 \\
Euglenophyta & 6 & 5 & 4 & 3 & 2 \\
Pyrrhophyta & 1 & 2 & 2 & 2 & 2 \\
Rodophyta & 0 & 1 & 1 & 1 & 1 \\
\hline \multicolumn{1}{c}{ Total } & $\mathbf{1 0 6}$ & $\mathbf{1 1 5}$ & $\mathbf{9 6}$ & $\mathbf{1 0 0}$ & $\mathbf{1 0 5}$ \\
\hline
\end{tabular}

Los resultados obtenidos por localidades, señalan que la riqueza total fue mayor en Miaría y menor en Kirigueti y Timpia. Por divisiones el mayor registro de Chlorophyta fue en Sepahua, el de Bacillariophyta en Timpia y el de Cyanophyta en Kirigueti y Miaría. Las Euglenophyta fueron más diversas en Sepahua (Tabla 3).

Durante las evaluaciones hubo fluctuaciones de la riqueza, relacionados con las épocas de vaciante y creciente. Sin embargo, entre localidades como Miaría y Timpía aunque se registra una leve tendencia al incremento de especies, la riqueza acumulada es muy diferente (Fig. 2).

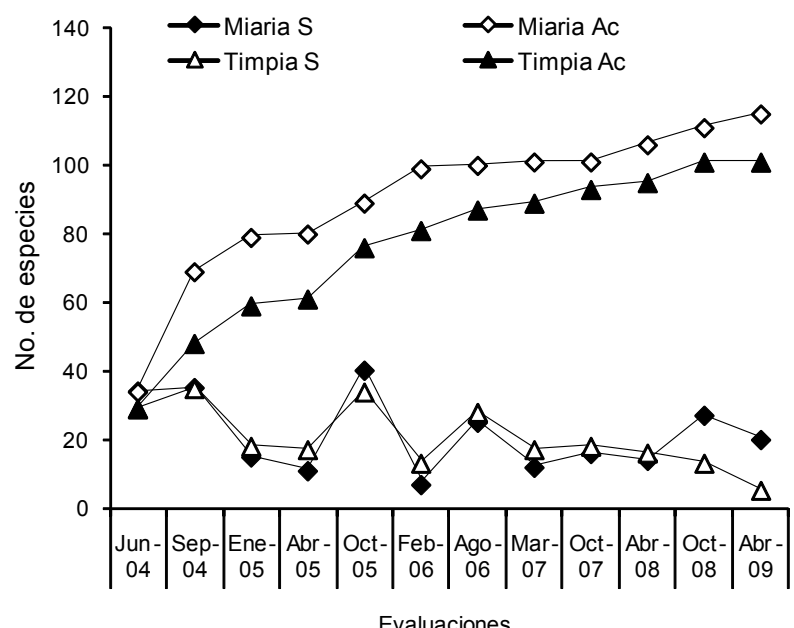

Figura 2. Riqueza (S) del plancton por evaluaciones en Miaria y Timpia. Bajo Urubamba. Junio 2004 - abril 2009.

\section{Bentos}

La riqueza total del bentos llega al registro de 112 especies, representando a 14 órdenes en tres phyla (Annelida, Arthropoda y Mollusca). Entre los ordenes son mas diversos los Trichoptera, seguido por Coleoptera y Ephemeroptera y resultando escasa la diversidad para los Oligochaeta, Megaloptera, Plecoptera y Unionoida (Tabla 4).

La mayor riqueza por órdenes fue registrada en Miaría y la menor en Timpia. Trichoptera, Coleoptera y Ephemeroptera fueron muy diversos en todas las localidades (Tabla 5). 
Tabla 4. Riqueza total del bentos por Ordenes. Bajo Urubamba. Setiembre 2003 - abril 2009.

\begin{tabular}{lc}
\hline \multicolumn{1}{c}{ ORDENES } & Riqueza total \\
\hline Oligochaeta & 1 \\
Decapoda & 3 \\
Coleoptera & 18 \\
Diptera & 13 \\
Ephemeroptera & 16 \\
Hemiptera & 12 \\
Lepidoptera & 2 \\
Megaloptera & 1 \\
Odonata & 13 \\
Orthoptera & 2 \\
Plecoptera & 1 \\
Trichoptera & 22 \\
Unionoida & 1 \\
Basomatophora & 7 \\
\hline \multicolumn{1}{c}{ Total } \\
\hline \multicolumn{1}{c}{}
\end{tabular}

Tabla 5. Riqueza (S) del bentos por Ordenes y localidades. Bajo Urubamba. Setiembre 2003 - abril 2009.

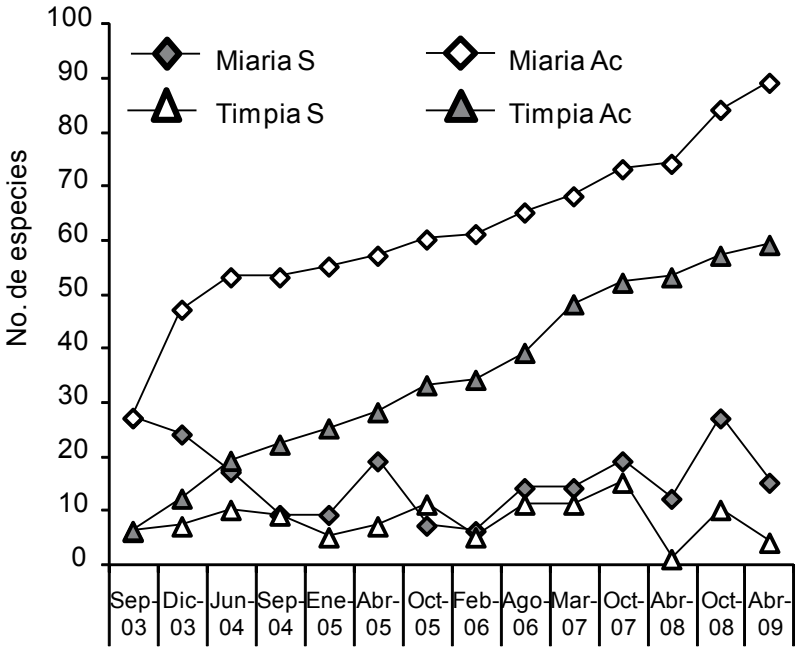

Evaluaciones

Figura 3. Riqueza (S) y riqueza acumulada (AC) del bentos por evaluaciones en Miaria y Timpia. Bajo Urubamba, Septiembre 2003 - abril 2009.

De acuerdo a las evaluaciones realizadas hubo fluctuaciones en la riqueza, relacionadas a las temporadas de vaciante y creciente. Destacándose que en las localidades de Miaría y Timpía se registra una leve tendencia al incremento de especies aunque la riqueza acumulada difiere notablemente (Fig. 3).

En relación al Índice Ephemeroptera + Plecoptera + Trichoptera (\%EPT), entre 2003 y 2009 aplicado en 51 de 210 evaluaciones, los valores fueron ubicadas en el rango superior (75 - 100) indicando la presencia de aguas de calidad optima y además, 41 evaluaciones $(50-75)$ indicando aguas de buena calidad (normal) (Tabla 6).

\section{Peces}

La riqueza total durante la evaluación fue de 176 especies, resultando mas diversos los Characiformes, y los Siluriformes, siendo escasamente representados los peces de origen marino: Myliobatiformes, Beloniformes y Synbranchiformes (Tabla 7).

Tabla 6. Valores del Indice Ephemeroptera + Plecoptera + Trichoptera (\%EPT) por evaluaciones y estaciones. Bajo Urubamba. Septiembre 2003 - abril 2009

\begin{tabular}{|c|c|c|c|c|c|c|c|c|c|c|c|c|c|c|c|}
\hline \multirow[b]{2}{*}{$\%$ EPT } & \multicolumn{3}{|c|}{ Sepahua } & \multicolumn{3}{|c|}{ Miaria } & \multicolumn{3}{|c|}{ Kirigueti } & \multicolumn{3}{|c|}{ Shivankoreni } & \multicolumn{3}{|c|}{ Timpia } \\
\hline & 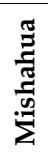 & 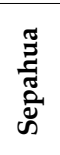 & 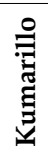 & 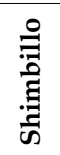 & 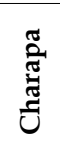 & $\underset{\Xi}{\stackrel{\Xi}{\Xi}}$ & 苟 & 它 & 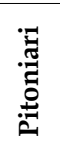 & 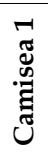 & 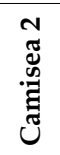 & 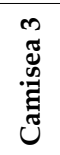 & 일 & 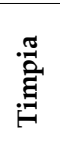 & 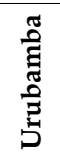 \\
\hline Sep-03 & 96 & 62 & 36 & 84 & 15 & 52 & 40 & 90 & 46 & 13 & 97 & 46 & 88 & 0 & 100 \\
\hline Dic-03 & 40 & 12 & 46 & 43 & 86 & 19 & 0 & 0 & 37 & 39 & 0 & 0 & 58 & 0 & 97 \\
\hline Jun-04 & 86 & 100 & 49 & 43 & 58 & 12 & 0 & 94 & 8 & 12 & 22 & 37 & 46 & 0 & 43 \\
\hline Sep-04 & 36 & 17 & 22 & 60 & 100 & 74 & 12 & 86 & 20 & 13 & 55 & 14 & 42 & 16 & 0 \\
\hline Ene-05 & 93 & 93 & 0 & 45 & 40 & 34 & 99 & 25 & 106 & 0 & 0 & 0 & 91 & 100 & 0 \\
\hline Abr-05 & 0 & 53 & 40 & 13 & 32 & 87 & 32 & 0 & 6 & 0 & 100 & 54 & 48 & 100 & 0 \\
\hline Oct-05 & 82 & 79 & 56 & 21 & 0 & 66 & 50 & 92 & 16 & 0 & 11 & 100 & 19 & 0 & 52 \\
\hline Feb-06 & 0 & 100 & 8 & 50 & 0 & 51 & 0 & 100 & 64 & 50 & 100 & 100 & 83 & 0 & 0 \\
\hline Ago-06 & 44 & 58 & 44 & 79 & 57 & 30 & 15 & 88 & 42 & 8 & 59 & 100 & 77 & 12 & 99 \\
\hline Mar-07 & 0 & 0 & 97 & 64 & 67 & 25 & 67 & 67 & 0 & 61 & 0 & 17 & 68 & 42 & 63 \\
\hline Oct-07 & 54 & 57 & 65 & 64 & 68 & 88 & 50 & 27 & 74 & 67 & 90 & 67 & 93 & 72 & 63 \\
\hline Abr-08 & 15 & 100 & 97 & 96 & 90 & 100 & 100 & 12 & 85 & 21 & 0 & 10 & 0 & 0 & 0 \\
\hline Oct-08 & 92 & 17 & 63 & 12 & 65 & 17 & 78 & 12 & 59 & 86 & 64 & 48 & 0 & 58 & 0 \\
\hline Abr-09 & 0 & 0 & 92 & 66 & 24 & 0 & 0 & 0 & 45 & 0 & 0 & 0 & 0 & 0 & 100 \\
\hline
\end{tabular}


Tabla 7. Riqueza total del necton por Ordenes. Bajo Urubamba. Septiembre 2003 - abril 2009.

\begin{tabular}{lc}
\multicolumn{1}{c}{ Ordenes } & Riqueza total \\
\hline Myliobatiformes & 1 \\
Beloniformes & 1 \\
Characiformes & 102 \\
Clupeiformes & 2 \\
Gymnotiformes & 4 \\
Perciformes & 8 \\
Pleuronectiformes & 2 \\
Siluriformes & 55 \\
Synbranchiformes & 1 \\
\hline \multicolumn{1}{c}{ Total } & $\mathbf{1 7 6}$ \\
\hline
\end{tabular}

Por localidades, la riqueza total fue mayor en Sepahua y Miaría y menor en Shivankoreni y Timpia.

Durante las evaluaciones hubo fluctuaciones de la riqueza, relacionados con las épocas de vaciante y creciente. Sin embargo, entre localidades como Miaría y Timpía, aunque se registra una leve tendencia al incremento de especies, la riqueza acumulada entre ambas localidades es muy diferente (Tabla 8, Fig. 4).

Sobre el Índice de Integridad Biológica (IBI). Los valores obtenidos durante la evaluación oscilan entre 33 y 52. Los mayores valores corresponden a Sepahua y Miaría, mientras que los menores se registran para Timpia. Por otro lado, los valores intermedios corresponden a Kirigueti y Shivankoreni. También fluctúan en relación a los periodos de vaciante y creciente (Tabla 9).

\section{Discusión}

Los ambientes acuáticos estudiados fueron en su mayoría lóticos, excepto la estación Laguna temporal (Kirigueti) que presenta características de aguas lénticas parte del año. En general, las temperaturas fueron similares; existen variaciones en las profundidades, transparencia y en el tipo de sustrato y
Tabla 8. Riqueza (S) del necton por Ordenes y localidades. Bajo Urubamba. Setiembre 2003 - abril 2009.

\begin{tabular}{|c|c|c|c|c|c|}
\hline 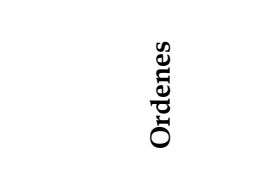 & 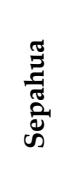 & 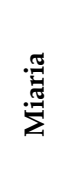 & 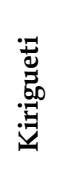 & 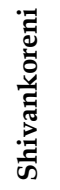 & $\stackrel{\widetilde{\Xi}}{\mathfrak{\Xi}}$ \\
\hline Myliobatiformes & 0 & 0 & 0 & 0 & 1 \\
\hline Beloniformes & 1 & 1 & 1 & 1 & 1 \\
\hline Characiformes & 76 & 74 & 57 & 44 & 49 \\
\hline Clupeiformes & 2 & 1 & 2 & 2 & 1 \\
\hline Gymnotiformes & 4 & 2 & 1 & 0 & 0 \\
\hline Perciformes & 7 & 7 & 2 & 3 & 0 \\
\hline Pleuronectiformes & 2 & 0 & 0 & 0 & 1 \\
\hline Siluriformes & 38 & 37 & 23 & 22 & 22 \\
\hline Synbranchiformes & 1 & 0 & 0 & 0 & 0 \\
\hline TOTAL & 131 & 122 & 86 & 72 & 75 \\
\hline
\end{tabular}

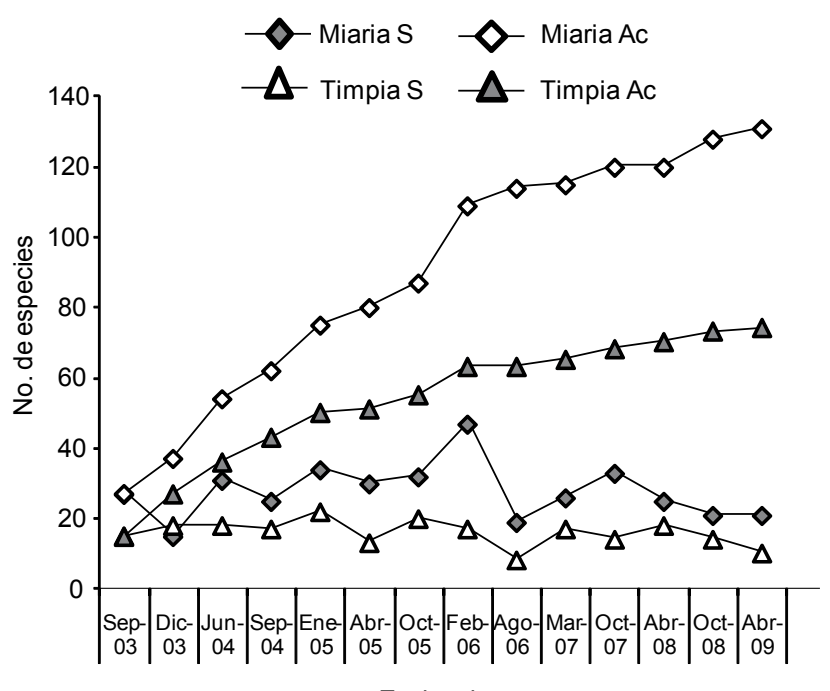

Evaluaciones

Figura 4. Riqueza (S) y riqueza acumulada (AC) del Necton por evaluaciones en Miaria y Timpia. Bajo Urubamba, Septiembre 2003 - abril 2009.

Tabla 9. Valores del Indice de Integridad Biologica (IBI) por evaluaciones y estaciones. Bajo Urubamba. Septiembre 2003 - abril 2009.

\begin{tabular}{|c|c|c|c|c|c|c|c|c|c|c|c|c|c|c|c|}
\hline \multirow[t]{2}{*}{ IBI } & \multicolumn{3}{|c|}{ Sepahua } & \multicolumn{3}{|c|}{ Miaria } & \multicolumn{3}{|c|}{ Kirigueti } & \multicolumn{3}{|c|}{ Shivankoreni } & \multicolumn{3}{|c|}{ Timpia } \\
\hline & 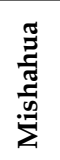 & 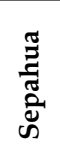 & 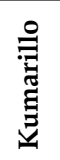 & 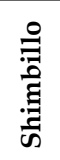 & 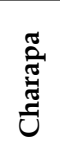 & $\underset{\Sigma}{\stackrel{\widetilde{J}}{\Sigma}}$ & $\frac{\pi}{\tilde{D}}$ & 幽 & 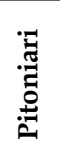 & 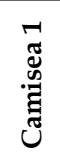 & 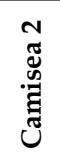 & 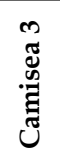 & $\begin{array}{l}\text { 열 } \\
\text { हี } \\
\text { कี }\end{array}$ & $\underset{\mathfrak{Z}}{\stackrel{a}{\Xi}}$ & $\begin{array}{l}\text { हू } \\
\text { हี } \\
\text { है } \\
\text { है }\end{array}$ \\
\hline Sep-03 & 44 & 44 & 48 & 48 & 50 & 44 & 40 & 46 & 40 & 48 & 46 & 50 & 38 & 34 & 38 \\
\hline Dic-03 & 40 & 44 & 44 & 44 & 50 & 46 & 46 & 44 & 46 & 46 & 44 & 46 & 40 & 36 & 36 \\
\hline Jun-04 & 44 & 42 & 46 & 48 & 48 & 42 & 36 & 36 & 44 & 40 & 42 & 36 & 50 & 32 & 32 \\
\hline Sep-04 & 52 & 48 & 48 & 42 & 38 & 52 & 48 & 50 & 40 & 46 & 48 & 42 & 40 & 40 & 36 \\
\hline Ene-05 & 52 & 50 & 52 & 44 & 40 & 44 & 52 & 46 & 36 & 44 & 42 & 46 & 40 & 36 & 36 \\
\hline Abr-05 & 52 & 44 & 50 & 44 & 38 & 52 & 42 & 40 & 42 & 44 & 40 & 38 & 38 & 42 & 34 \\
\hline Oct-05 & 52 & 42 & 50 & 46 & 42 & 50 & 40 & 42 & 34 & 44 & 46 & 38 & 40 & 38 & 40 \\
\hline Feb-06 & 50 & 44 & 48 & 42 & 48 & 44 & 40 & 38 & 34 & 42 & 36 & 38 & 36 & 36 & 30 \\
\hline Ago-06 & 44 & 42 & 42 & 40 & 44 & 38 & 42 & 46 & 32 & 40 & 42 & 38 & 34 & 36 & 32 \\
\hline Mar-07 & 50 & 32 & 48 & 46 & 48 & 44 & 38 & 38 & 40 & 30 & 42 & 38 & 44 & 34 & 44 \\
\hline Oct-07 & 46 & 42 & 43 & 40 & 38 & 38 & 35 & 37 & 39 & 38 & 43 & 42 & 40 & 38 & 38 \\
\hline Abr-08 & 40 & 33 & 39 & 39 & 35 & 31 & 31 & 34 & 34 & 39 & 31 & 35 & 37 & 32 & 30 \\
\hline Oct-08 & 43 & 52 & 44 & 41 & 45 & 47 & 36 & 36 & 37 & 42 & 44 & 41 & 37 & 40 & 36 \\
\hline Abr-09 & 42 & 37 & 38 & 48 & 39 & 44 & 39 & 40 & 29 & 39 & 40 & 29 & 44 & 36 & 36 \\
\hline
\end{tabular}


diferencias más notorias en relación al declive y la ubicación geográfica (altitud) de las localidades en evaluación. Timpía y Shivankoreni, ubicadas aguas arriba presentaron ambientes con mayor declive y torrencialidad, mientras que Sepahua y Miaria, aguas abajo, presentaron ambientes con menor declive y caudal con velocidad moderada a lenta, lo cual podría sustentar la existencia de mayor variedad de hábitats acuáticos, aspecto también conocido como mayor heterogeneidad de espacio y recursos (Welcomme 1985).

La diversidad registrada del plancton se sustenta en la variedad de Chlorophyta (algas verdes), Bacillariophyta (diatomeas) y Cyanophyta (algas azul-verdes), aun cuando la transparencia fue mínima y marcada velocidad en la mayoría de ambientes acuáticos evaluados. Destacando como mas diversos los ambientes de Miaría y Sepahua, probablemente por la mayor transparencia en las quebradas y existencia de mayor variedad de hábitats, confirmado por la riqueza acumulada en Miaría y Timpía. En Chlorophyta el resultado fue cercano al registrado en Pucallpa (Samanez 1979).

En el bentos, la mayor riqueza la presentó Arthropoda, dominada por larvas y adultos de insectos acuáticos. Entre localidades, una mayor riqueza en Miaría por presentar mayor heterogeneidad y la menor en Timpia. Entre los ordenes de insectos registrados destacan: Trichoptera, Coleoptera y Ephemeroptera.

En el necton, la riqueza total es comparable al registrado en el Bajo Río Huallaga (Ortega et al. 2007) se basa en la diversidad de peces con escamas (Characiformes), y los peces de cuero (Siluriformes), como sucede en distintos lugares de la Amazonia (Ortega et al. 2007, Goulding et al. 2004) y la mayoría de tamańo menudo a mediano. La mayor diversidad observada en Sepahua tendría relación directa con la mayor heterogeneidad de hábitats y la disponibilidad de recursos favorables en la parte baja del sistema estudiado.

Entre las microalgas, las diatomeas son indicadoras de calidad de sistemas lóticos, pero exige un conocimiento al nivel de especies para ser aplicado (Lobo et al. 2004).

Los valores encontrados de \%EPT indicaron aguas de muy buena calidad en 51 estimaciones (24\%), y en 44 evaluaciones (21\%) confirman aguas normales. Por otro lado, considerando que los valores mínimos fueron registrados en la época lluviosa, cuando es difícil localizar los insectos indicadores, los valores de $\%$ EPT obtenidos no implicarían necesariamente que la calidad de las aguas fuera muy distinta a la normal.

En cuanto al estado de conservación basado en los peces y los valores del IBI, los valores obtenidos por localidad y por evaluación demuestran que en el Bajo Urubamba, especialmente en Miaría y Sepahua, existen condiciones entre buena y muy buena, que demuestra mejores condiciones de conservación que los obtenidos para los ambientes acuáticos evaluados entre Tarapoto y Yurimaguas (Ortega et al. 2007).

\section{Agradecimientos}

Esta evaluación, ha sido posible gracias a la colaboración importante de B. Rengifo, M. Velásquez, R. Quispe, V. Palacios, Isabel Corahua y Alex Mendoza; miembros del Departamento de Ictiología del MHN-UNMSM. Nuestro especial reconocimiento a los Gerentes de ERM PERU S.A. por las facilidades recibidas y a PLUSPETROL por el auspicio de la investigación.

\section{Literatura citada}

Barthem, R., M. Goulding, B. Forsberg, C. Cañas, H Ortega. 2004. Ecología Acuática. Bases Científicas para la Conservación de Cabeceras Andino-Amazónicas. Asociación para la Conservación de la Cuenca Amazónica (ACCA). Gráfica Biblos S. A. Lima Perú. 117 pp.

Drouet, F and W.A. Daily. 1966. The Catherwood Foundation Peruvian Amazon. Cyanophyta. Monograph. Acad. Nat. Sci. Phila. Philadelphia (14):445-448.

De Rham P., M. Hidalgo, \& H. Ortega. 2001. Los Peces del BiaboCordillera Azul. En: "Perú: Biabo Cordillera Azul", Rapid Biological Inventories Report 2. Alverson, W.S., L.O. Rodríguez \& D. Moskovits (eds.). Chicago, IL: The Field Museum.

Dudgeon, D., A. Arthington, M. Gessner, Z. Kawabata, D. Knowler; C. Leveque, R. Naiman. A. Prieur-Richard, D. Soto, M. Stiassny \& C. Sullivan. 2005. Freshwater Diversity: Importance, threats, status and conservation challenges. (www.diversitas.org/freshwater)

Goulding, M., R. Barthem, C. Cañas, B. Forsberg, H. Ortega. 2004. AMAZON HEADWATERS Rivers, Wildlife and Conservation in the Southeastern Peru. ACA, ACCA, Lima. 198 pp.

Karr, J.R. 1981. Assessment of Biotic Integrity using fish communities. Fisheries, 6(6): $21-26$.

Lévêque, C., T. Oberdorff, D. Paugy, M. L. J. Stiassny, y P. A. Tedesco. 2007. Global diversity of fish (Pisces) in freshwater. Hydrobiologia 595(1): 545-567. doi:10.1007/ s10750-007-9034-0.

Lobo, E.A.; V.L.M. Callegaro; G. Hermany; D. Bes; C.A. Wetzel \& M.A. Oliveira. 2004. Use of epilithic diatoms as bioindicators from lotic systems in southern Brazil, with special emphasis on eutriphication Acta Limnol. Bras., 16(1)25-40.

Ortega, H. 1996. Evaluación preliminar de la Ictiofauna del río Camisea, Camisea, La Convención, CUSCO, PERU. In Proceedings from the Workshop on Biological and Cultural Diversity of the Lower Urubamba, Peru. Biodiversity Program, Smithsonian Institution, Washington, DC. pp 83-90.

Ortega, H., I. Samanez, E. Castro, M. Hidalgo y N. Salcedo. 1998. Protocolos Sugeridos para la Evaluación y el Monitoreo de los Sistemas Acuáticos del Bajo Urubamba, Perú. Biodiversity Assessment \& Monitoring, Smithsonian Institution/ MAB Series \#2: 278-280.

Ortega, H., M. Hidalgo, N. Salcedo, E. Castro y C. Riofrío. 2001. Diversity and Conservation of Fish of the Lower Urubamba Region, Peru. 143-150 p. En: Urubamba: Biodiversity of a Peruvian Rainforest. Alonso, A., F. Dallmeier and P. Campbell, eds. 2001. SI/MAB Series \#7. Smithsonian Institution, Washington, D.C.

Ortega, H., M. Hidalgo. 2008. Freshwater fishes and Aquatic habitats in Peru: Current knowledge and conservation. Aquatic Ecosystem Health \& Management. Vol.11 (3):257-272. Taylor and Francis Group.

Ortega, H., M. Hidalgo y G. Bertiz. 2003. Peces del río Yavarí. En: Pitman, N., C. Vriesendorp, D. Moskovits (eds.). Perú: Yavarí. Rapid Biological Inventories Report 11. Chicago, IL: The Field Museum.

Ortega H., B. Rengifo, I. Samanez \& C. Palma. 2007. Diversidad y el estado de conservación de cuerpos de agua Amazónicos en el nororiente del Perú. Rev. peru. biol. 13(3): 189 - 193

Reis, R., S. O. Kullander \& C. J. Ferraris Jr. 2003. Check List of the Freshwater fishes of South and Central America. Pontifícia Universidade Católica do Río Grande do Sul. EDIPUCRS Brasil. 985 pp. 
Roldán, G. 1999. Macroinvertebrados y su valor como indicadores de la calidad de agua. Revista Academia Colombiana de Ciencias Exactas, Físicas y Naturales. Vol. 23(88) 375-387.

Salcedo, N. M. Hidalgo, P. Minaya, E. Castro, R. Acosta, D. Reyes, J. León and S. Udvardy. 2001. Biodiversity Assessment of the Aquatic Ecosystems of the Lower Urubamba Region, Peru. Pp: 37-47. En: Urubamba: Biodiversity of a Peruvian Rainforest. Alonso, A., F. Dallmeier and P. Campbell, eds. 2001. SI/MAB Series \#7. Smithsonian Institution, Washington, D.C.

Samanez, I. 1979. Algas Continentales del Perú II. Algas de la Zona de Pucallpa y Alrededores. Bol. Bot. Mus. Hist. nat. UNMSM.

Samanez, I. 1988. Rotíferos Planctónicos de la Amazonía Peruana I. Departamento de Ucayali, Perú. Rev. Per. Biol. (1)141-167.

Samanez, I. 1991. Rotíferos Planctónicos de la Amazonía Peruana II: Departamento de Loreto. Publ. Mus. Hist. nat. (A) 38:1-4.
Samanez, I. \& C. Riofrio, 1995. Composición de la fauna de Rotíferos y su relación con las macrofitas acuáticas en una laguna fluvial, Ucayali. Publ. Mus. Hist. nat. (A)50:20-30.

Samanez, I. \& F. Zambrano. 1995. Observaciones sobre la Diversidad y Algunas Características del Plancton en el Dpto. Madre de Dios, Perú. Publ. Mus. Hist. nat. (A) 51:1-12.

Samanez, I. 2002. La fauna de Rotífera y las macrofitas en las lagunas Cashibo y Yarina, Departamento de Ucayali, Perú. Tesis Maestría en Recursos Acuáticos, mención - Ecología Acuática, FCB, UNMSM. Lima. 68p.

Riofrio, J., I. Samanez, F. Carrasco y M. Clavo, 2003. Caracterización Limnológica de la Laguna Cashibococha (Ucayali-Perú) durante 2001. Rev. per. Biol. 2003; 10 (2): 183 - 194.

Welcomme, R. L. 1985. River Fisheries. FAO Fish. Tech. Pap., (26): $330 \mathrm{pp}$. 


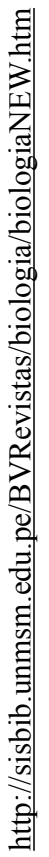

